\title{
Editorial
}

François A. Béique MD FRCPC Josée Lavoie MD FRCPC

\section{TEE Monitoring}

volaemia and a decrease in LVEDA. ${ }^{8}$ In the study of Leung $e t a l$, the presence of left ventricular end systolic cavitary obliteration was associated with hypovolaemia in $80 \%$ of patients after cardiac surgery. ${ }^{9}$ Bergquist $\varepsilon t$ al found that intraoperative hypovolaemia was identified predominantly with TEE in $30 \%$ of patients while the PAC was only useful in establishing this diagnosis in $7 \%$ of cardiac surgical patients. ${ }^{10}$ Using the Frank-Starling curve Thys $e t$ al found poor correlation between PAOP and cardiac index (CI) while LVEDA measurements had a strong correlation with CI. ${ }^{11}$ Right ventricular filling volume is more difficult to assess because of its crescent shape. Although there is no consensus on measurements of right ventricular diameter, an increase or a decrease of the same diameter provides useful information on changes in preload condition. The normal range of right and left ventricular diameters have been well established with TTE in adults but is not available for children. ${ }^{12}$ This does not invalidate the usefulness of the technique in paediatric patients because the trend during postural and haemodynamic changes is frequently more useful than an isolated single measurement.

Monitoring of preload should include the interrogation of mitral and pulmonary venous inflow with pulsed wave Doppler. The systolic component of pulmonary venous flow occurs when the mitral valve is closed and is inversely related to left atrial filling pressure. Pulmonary venous diastolic flow occurs when the mitral valve is opened and, therefore, provides information on the filling pressure of the left ventricle. Analysis of mitral inflow provides further insight on preload conditions. Variation in the ratio of mitral $\mathrm{E}$ velocity which represents early ventricular filling and $A$ velocity during atrial systole correlates with PAOP. ${ }^{13} \mathrm{~A}$ comparison of forward mitral atrial flow (A wave) with reverse pulmonary venous flow (AR wave) during atrial systole is also a good indicator of the relative impedance to blood flow at those two sites and correlates with PAOP. ${ }^{14}$ The interpretation of pulmonary venous

From the Department of Anaesthesia, the Sir Mortimer B. Davis Jewish General Hospital and Montréal Childrens Hospital, McGill University, Montréal, Canada

Address correspondence to: Dr. François A. Béïque MD FRCPC, Department of Anaesthesia, Sir Mortimer B Davis Jewish General

Hospital, 3755 Chemin de la Côte St-Catherine, Montréal, Canada H3T 1E2

Acknowledgement: special thanks to Dr J-F Courval for reviewing this manuscript 
flow and mitral inflow provide valuable insight on preload conditions but this data may not be reliable in patients with diastolic dysfunction and severe pulmonary disease. The interatrial septum can also be used as an indicator of volume status and cardiac dysfunction because it is a thin structure that will respond to changes in left and right atrial pressure. ${ }^{15}$

\section{Global and regional cardiac function}

\section{Global cardiac function}

Diastolic function can be evaluated with Doppler interrogation of mitral inflow pattern and pulmonary venous flow. ${ }^{16}$ Methods of evaluating left ventricular systolic function include, but are not limited to: fractional area change from LVEDA and LVESA measurement, fractional shortening, speed of fractional shortening, descent of the base of the left ventricle and Doppler measurement of cardiac output. ${ }^{5}$ Ejection fraction can be calculated from the end systolic and end diastolic volumes. ${ }^{5}$ Load independent assessment of cardiac function can also be obtained by constructing an end systolic pressure volume curve with the measurement of arterial pressure and ventricular filling volume or area as previously described by Gorcsan. ${ }^{17}$ Right ventricular ejection fraction based on volume or area change is difficult to obtain because of the crescent shape of the right ventricle. Other methods of evaluating right ventricular function include descent of the base of the heart at the level of the tricuspid valve, ${ }^{18}$ right ventricular long or short axis shortening in the four chamber view ${ }^{18}$ and Doppler interrogation of the inferior vena cava. ${ }^{19}$

\section{Regional cardiac function}

In the evaluation of regional cardiac function, echocardiography is a sensitive monitor for myocardial ischaemia. In contrast, only one third of ischaemic episode are detected by the PAC as an elevation in PAOP. ${ }^{20}$ When compared with electrocardiographic monitoring, regional wall motion abnormality occurs up to ten minutes earlier after partial coronary occlusion in dogs. ${ }^{21}$ Wall thickening is a more specific indicator of ischaemia than wall motion anomaly. Other factors can create wall motion abnormality without ischaemia including changes in loading conditions, conduction abnormalities, old myocardial infarction (MI), rotation and translation of the heart axis. The most commonly used view to monitor for myocardial ischaemia is the transgastric mid-papillary view because it images myocardium that is supplied by all three coronary arteries. Multiple views should be used, otherwise ischaemic episodes at the base and at the apex will be missed. The addition of a longitudinal plane will increase detection of myocardial ischaemia by approximately $35-40 \% .{ }^{22-23}$ Leung et al demonstrated that new regional wall motion abnormality after cardiopulmonary bypass was associated with adverse outcome without ECG changes in 6 of 18 patients. ${ }^{24}$ Similarly, Comunale $e t a l$ also found that TEE was twice as predictive as ECG in identifying patients with MI. ${ }^{25}$ Contrary to these studies, others found that TEE had little incremental value in identifying patients at high risk for perioperative ischaemic outcomes but only the transgastric mid-papillary view was monitored. ${ }^{26-28}$

\section{Abnormal cardiac blood flow}

Category one indications in patients with abnormal cardiac blood flow include the evaluation of mitral valve repair, complex congenital cardiac surgery and is invaluable in the evaluation of an unstable patient with unexplained haemodynamic instability. ${ }^{1,2}$ Transoesophageal echocardiography not only provides information on preload and cardiac function but also allows prompt evaluation of other pathologies including ventricular septal defect, ruptured papillary muscle, ischaemic mitral regurgitation and aortic dissection. ${ }^{1}$

\section{Limitations of TEE}

Transoesophageal echocardiography is an important monitoring and diagnostic tool but, despite the wave of enthusiasm, there are limitations to its use. The cost of the equipment can vary from $\$ 100,000$ to $>\$ 300,000$ for state of the art technology. The development of sufficient expertise in TEE also requires a considerable amount of training before proficiency is achieved. The American Society of Echocardiography recommends six months of training to achieve proficiency at an advanced level. ${ }^{29}$ Poor imaging may also hinder a full cardiovascular assessment. In addition, the innominate artery and part of the ascending aorta are shadowed by the right main stem bronchus and or the trachea. Only two of the three great arch vessels can be visualized in $60 \%$ of patients which limits TEE evaluation of this area of the thoracic aorta in trauma or suspected dissection. ${ }^{3}$ The PAC has certain advantages. In the evaluation of a patient with cardiogenic shock or a low output state it enables us to measure mixed venous oxygen saturation and to calculate oxygen delivery and consumption. In addition, the PAC provides direct and continuous measurement of intravascular and intracardiac pressures.

\section{Conclusion}

The role of TEE is continuously reevaluated: as we achieve more experience new indications for TEE are discovered. It is important, as we study haemodynamic 
changes, that we take full advantage of the diagnostic and monitoring capabilities of TEE. In the study of Soliman $e t a l$ it is unfortunate that no measurements of right ventricular function and preload were obtained. ${ }^{4}$ Sequential measurements of right atrial and ventricular diameter would have established the presence of an increase or a decrease in filling volume of right sided chamber when positioning the patient prone. Doppler interrogation of mitral inflow and pulmonary venous flow should be routinely performed and reported, especially in a study where cardiac filling volume and function are evaluated. Transoesophageal echocardiography is an important diagnostic tool if used properly and only with rigorous cardiovascular assessment can we hope to obtain the answers we are looking for. The role of TEE will ultimately depend on outcome studies and whether the added cost is justified by cost-benefit analysis.

\section{Le monitorage par ETO}

L'échocardiographie a été utilisée d'abord en 1954 et, avec l'évolution de l'imagerie cardiaque bidimensionnelle (BD) en 1972, l'échocardiographie transthoracique est devenue un important outil de diagnostic clinique. C'est la mise au point d'une sonde d'échocardiographie transosophagienne (ETO) à haute résolution en 1989 qui a permis le développement rapide du rôle de l'ETO periopératoire. Chez le sujet souffrant d'instabilité hémodynamique inexpliquée, l'ETO contribue souvent à l'amélioration de l'évolution clinique. ${ }^{1}$ Elle fournit des informations plus exactes que le cathéter de l'artère pulmonaire (CAP) sur la précharge, la fonction cardiaque et les anomalies du débit cardiaque. $^{2}$ Ses autres applications comprennent son emploi dans la détection des embolies et dans l'évaluation d'une atteinte athérosclérotique de l'aorte. ${ }^{3}$ L'étude menée par Soliman et coll. montre le rôle étendu de l'ETO en tant que moniteur pour des patients souffrant de pression veineuse centrale élevée et devant subir la correction d'une cyphoscoliose. ${ }^{4}$ Cette étude présente des limites, comme le soulignent ses auteurs, qui concernent plus précisément l'évaluation systématique et approfondie, à l'aide de l'ETO, de paramètres cardiovasculaires dont nous allons maintenant parler.

\section{Précharge}

L'échocardiographie transœsophagienne permet de visualiser le volume de remplissage des deux ventricules cardiaques, le gauche et le droit. Le remplissage du ven- tricule gauche est souvent mesuré en traçant par planimétrie l'aire télodiastolique et l'aire télosjstolique du ventricule gauche (ATDVG, ATSVG) sur un axe court au niveau mésopapillaire. En variante, on peut aussi procéder à une mesure acoustique continue de l'aire du ventricule gauche et à la visualisation de chaque cycle cardiaque. La mesure du diamètre ventriculaire peut aussi servir à estimer le volume de remplissage. ${ }^{5}$ Dans la plupart des études, il y a une bonne corrélation entre l'estimation du remplissage ventriculaire avec radionucléide et celle avec ETO, mais une faible corrélation avec la pression artérielle pulmonaire diastolique ou la pression artérielle pulmonaire bloquée (PAPB) $\cdot^{6,7}$ En outre, il y a une corrélation linéaire entre l'hypovolémie progressive et une diminution de l'ATDVG. ${ }^{8}$ Dans l'étude de Leung et coll., la présence d'une oblitération cavitaire télosystolique du ventricule gauche était associée à une hypovolémie chez $80 \%$ des patients qui avaient subi une chirurgie cardiaque. ${ }^{9}$ Bergquist et coll. on trouvé que l'hypovolémie peropératoire a été identifiée surtout avec l'ETO chez $\mathbf{3 0} \%$ des patients alors que le CAP n'a permis d'établir ce diagnostic que chez $7 \%$ des patients de chirurgie cardiaque. ${ }^{10}$ En utilisant la courbe de Frank-Starling, Thys et coll. n'ont trouvé qu'une faible corrélation entre la TAPB et l'index cardiaque (IC) tandis que les mesures de l'ATDVG présentaient une forte corrélation avec l'IC." Le volume de remplissage du ventricule droit est plus difficile à évaluer à cause de sa forme en croissant. Bien qu'on n'ait pas établi une règle générale de mesure du diamètre du ventricule droit, une augmentation ou une diminution du même diamètre renseigne utilement sur les modifications survenant lors de la précharge. L'étendue normale des diamètres ventriculaires droit et gauche a été clairement déterminée selon l'ETO chez les adultes, mais non chez les enfants. ${ }^{12}$ L'utilité de la technique n'est pas pour autant invalidée auprès des patients pédiatriques parce que la tendance des mesures pendant les changements posturaux et hémodynamiques est souvent plus utile qu'une mesure unique isolée.

Le monitorage de la précharge devrait inclure le sondage des régions de remplissage mitral et veineux pulmonaire avec des ondes Doppler pulsées. La composante systolique du débit veineux pulmonaire se manifeste lorsque la valve mitrale se ferme et cette composante est inversement reliée à la pression de remplissage auriculaire gauche. Le remplissage veineux pulmonaire diastolique se produit lorsque la valve mitrale s'ouvre et, donc, nous informe sur les pressions de remplissage du ventricule gauche. L'analyse du remplissage mitral fournit une meilleure compréhension des conditions de précharge. Les variations du rapport de la vélocité mitrale $\mathrm{E}$, représentant le début du remplissage 
ventriculaire, et de la vélocité $A$, durant la systole auriculaire, est corrélé avec la PAPB. ${ }^{13}$ Une comparaison du débit mitral frontal (onde $A$ ) avec le débit veineux pulmonaire refoulé (onde AR), pendant la systole auriculaire, est aussi une bonne mesure de l'impédance relative du flot sanguin à ces deux sites et est aussi corrélée avec la PAPB ${ }^{14}$ L'interprétation du débit veineux pulmonaire et du débit de remplissage mitral amène à une meilleure compréhension des conditions de précharge, mais ces données peuvent ne pas être fiables chez les patients souffrant de dysfonction diastolique et de maladie pulmonaire sévère. Le déplacement du septum interauriculaire peut aussi être utilisé comme un indicateur de la volémie et de la dysfonction cardiaque, puisque le septum est une structure mince réagissant aux changements de pression auriculaire droite et gauche. ${ }^{15}$

\section{Fonction cardiaque globale et régionale}

\section{Fonction cardiaque globale}

La fonction diastolique peut être évaluée par sondage Doppler de la morphologie du remplissage mitral et des débits veineux pulmonaires. ${ }^{16}$ Les méthodes d'évaluation de la fonction systolique ventriculaire gauche incluent, sans s'y limiter : le changement de l'aire fractionnaire en utilisant les mesures de l'ATDVG et de l'ATSVG, le raccourcissement fractionnaire, la vitesse du raccourcissement fractionnaire, l'abaissement de la base du ventricule gauche et la mesure du débit cardiaque par écho Doppler. ${ }^{5} \mathrm{La}$ fraction d'éjection peut être chiffrée au moyen des volumes télosystoliques et télodiastoliques. ${ }^{5}$ Une évaluation de la fonction cardiaque indépendante des charges peut être obtenue en construisant une courbe volume-pression télosystolique à partir de la mesure de la tension artérielle et le volume ou l'aire de remplissage ventriculaire ainsi que le décrit Gorcsan. ${ }^{17} \mathrm{Le}$ calcul de la fraction d'éjection du ventricule droit, basé sur le volume ou l'aire, est difficile à réaliser étant donné la forme en croissant du ventricule droit. D'autres méthodes pour évaluer la fonction ventriculaire droite incluent : l'abaissement de la base du coeur au niveau de la valve tricuspidienne, le raccourcissement des axes longs et courts du ventricule droit dans un plan échographique des quatre chambres ${ }^{18}$ et le sondage par écho Doppler de la région de la veine cave inférieure. ${ }^{19}$

\section{Fonction cardiaque régionale}

Dans l'évaluation de la fonction cardiaque régionale, l'échocardiographie est un moniteur sensible à l'ischémic myocardique. Par contre, un tiers seulement des épisodes ischémiques sont détectés par le CAP sous la forme d'une élévation de la PAPB. Quand on les compare à l'aide du monitorage électrocardio- graphique, les anomalies régionales de la paroi ventriculaire se manifestent jusqu'à dix minutes plus précocément après une occlusion partielle coronaire chez les chiens. ${ }^{21}$ L'épaississement de la paroi est un indicateur plus précis de l'ischémie qu'une anomalie du mouvement de la paroi. D'autres facteurs peuvent créer une anomalie du mouvement de la paroi sans ischémie : les changements des conditions de charge, des anomalies de conduction, un ancien infarctus du myocarde (IM), la rotation et la translation de l'axe cardiaque. L'incidence la plus fréquemment utilisée pour surveiller l'ischémie myocardique est le plan de coupe transgastrique mésopaillaire qui illustre le myocarde nourri par les trois artères coronaires. Il faut utiliser différents plans de coupe, autrement les épisodes ischémiques survenant à la base et à l'apex ne seront pas décelés. L'ajout d'un plan longitudinal augmentera la détection de l'ischémie myocardique d'environ $35-40 \%{ }^{22,23}$ Leung et coll. ont démontré qu'une nouvelle anomalie de mouvement de la paroi, à la suite d'une circulation extracorporelle, était associée à des effets secondaires sans changements de l'ECG chez 6 des 18 patients de l'expérience. ${ }^{24}$ De même, Comunale et coll. ont aussi montré que l'ETO présente une valeur prédictive deux fois meilleure à celle de l'ECG dans l'identification de l'IM. ${ }^{25}$ Contrairement à ce que ces études révèlent, d'autres chercheurs ont trouvé que l'ETO présentait une valeur incrémentielle mineure lors de l'identification des patients à haut risque de conséquences ischémiques périopératoires, mais seule l'incidence transgastrique mésopapillaire avait été examinée. ${ }^{26-28}$

\section{Anomalie du débit coronarien}

Les indications de première catégorie chez les sujets souffrant d'anomalie du débit coronarien comprennent l'évaluation de la réparation de la valvule mitrale, la chirurgie cardiaque congénitale complexe et l'ETO est d'un grand secours dans l'évaluation d'un patient qui présente une instabilité hémodynamique inexpliquée. $^{2}$ L'échocardiographie transosophagienne fournit non seulement des informations sur la précharge et la fonction cardiaque, mais elle permet également l'évaluation rapide d'autres pathologies comprenant la communication interventriculaire, la rupture du muscle papillaire, l'insuffisance mitrale ischémique et la dissection aortique.

\section{Limites de l'ETO}

L'échocardiographie transœsophagienne est un outil important de monitorage et de diagnostic, mais en dépit d'une vague d'enthousiasme, son emploi comporte certaines limites. En effet, le coût de l'équipement varie de 
100000 \$ à plus de $300000 \$$ pour une technologie de pointe. Le développement d'une expertise suffisante dans le domaine de l'ETO exige aussi de la compétence, fruit d'une longue formation. L'American Society of Echocardiography (Société américaine d'échocardiographie) recommande six mois de formation pour atteindre un degré avancé de compétence. ${ }^{29}$ Une imagerie déficiente peut aussi empêcher une évaluation cardiovasculaire complète. De plus, le tronc artériel brachiocéphalique et une partie de l'aorte ascendante sont masqués par la bronche souche droite et/ou par la trachée. Seulement deux des trois grands arcs vasculaires peuvent être visualisés chez $60 \%$ des patients, ce qui limite l'évaluation ETO de cette portion de l'aorte thoracique dans les cas de traumatisme ou de dissection appréhendée. ${ }^{3}$ Le CAP offre certains avantages. Lors de l'évaluation d'un patient présentant un choc cardiogénique ou une insuffisance antérograde, il permet la mesure de la saturation en oxygène du sang veineux mêlé et le calcul de la livraison et de la consommation d'oxygène. Le CAP fournit aussi une mesure directe et continue des pressions intravasculaire et intracardiaque.

\section{Conclusion}

Le rôle de l'ETO est réévalué continuellement : à mesure que nous acquérons de l'expérience, nous découvrons de nouvelles indications. Il est important, alors que nous étudions les changements hémodynamiques, de tirer tous les avantages des possibilités de diagnostic et de monitorage de l'ETO. Dans l'étude de Soliman $e t$ coll., il est dommage qu'aucune mesure de la fonction du ventricule droit et de la précharge n'ait été obtenue. ${ }^{4}$ Des mesures séquentielles du diamètre de l'oreillette et du ventricule droits auraient déterminé la présence d'une augmentation ou d'une diminution du volume de remplissage de la cavité droite lorsque le patient est installé en décubitus ventral. Le sondage Doppler du remplissage mitral et du débit de la veine pulmonaire devraient être des examens de routine et on devrait en faire mention, surtout dans une étude où le volume et la fonction de remplissage cardiaque sont évalués. L'échocardiographie transœsophagienne est un important outil diagnostique s'il est correctement utilisé, et ce n'est qu'avec une évaluation cardiovasculaire rigoureuse que nous pouvons espérer obtenir les réponses que nous cherchons. Le rôle de l'ETO va dépendre ultimement des études de résultats et aussi de la justification des coûts additionnels déterminés par une analyse coût-bénéfices.

\section{References}

1 Practice guidelines for perioperative transesophageal echocardiography: A report by the American Society of Anesthesiologists and the Society of Cardiovascular
Anesthesiologists Task Force on Transesophageal Echocardiography. Anesthesiology 1996; 84: 986-1006.

2 Reichert CL, Visser CA, Koolen J], et al. Transesophageal echocardiography in hypotensive patients after cardiac operations. Comparison with hemodynamic parameters. J Thorac Cardiovasc Surg 1992; 104: 321-6.

3 Béique FA, Joffe D, Tousignant $G$, Konstadt S. Echocardiography-based assessment and management of atherosclerotic disease of the thoracic aorta. J Cardiothorac Vasc Anesth 1998; 12: 206-20.

4 Soliman DE, Maslow AD, Bokesch PM, et al. Transesophageal echocardiography during scoliosis repair. Can J Anaesth 1998; 45: 925-932

5 Aurigemma GP, Douglas PS, Gaasch WH. Quantitative evaluation of left ventricular structure wall stress, and systolic function. In: Otto CM. The Practice of Clinical Echocardiography. Philadelphia: WB Saunders, 1997: 1-24.

6 Harpole DH, Clements FM, Quill T, Wolfe WG, Jones $R H, M c C a n n R L$. Right and left ventricular performance during and after abdominal aortic aneurysm repair. Ann Surg 1989; 209: 356-62.

7 Ryan T, Burwash I, Lu J, et al. The agreement between ventricular volumes and ejection fraction by transesophageal echocardiography or a combined radionuclear and thermodilution technique in patients after coronary artery surgery. J Cardiothorac Vasc Anesth $1996 ; 10: 323-8$.

8 Cheung AT, Savino JS, Weiss SJ, Aukburg SJ, Berlin JA. Echocardiographic and hemodynamic indexes of left ventricular preload in patients with normal and abnormal ventricular function. Anesthesiology 1994; 81: 376-87.

9 Leung JM, Levine EH. Left ventricular end-systolic cavity obliteration as an estimate of intraoperative hypovolemia. Anesthesiology 1994; 81: 1102-9.

10 Bergquist BD, Bellows WH, Leung JM. Transesophageal echocardiography in myocardial revascularization: II. Influence on intraoperative decision making. Anesth Analg 1996; 82: 1139-45.

11 Thys DM, Hillel Z, Goldman ME, Mindich BP, Kaplan JA. A comparison of hemodynamic indices derived by invasive monitoring and two-dimensional echocardiography. Anesthesiology 1987; 67: 630-4.

12 Triulzi $M$, Gillam LD, Gentile $F$, Newell JB, Weyman $A E$. Normal adult cross-sectional echocardiographic values: linear dimensions and chamber areas. Echocardiography. A Review of cardiovascular ultrasound 1984; 1: 403-20.

13 Samuelsson S, Brodin LA, Broman M, Ovall $A$, Settergren $G$. Comparison between transesophageal Doppler echocardiography and nuclear cardioangiography for the evaluation of left ventricular filling during coronary artery bypass grafting. Anesth Analg 1995; 80: 41-6. 
14 Rossvoll $O$, Hatle LK. Pulmonary venous flow velocities recorded by transthoracic Doppler ulrasound: relation to left ventricular diastolic pressure. J Am Coll Cardiol 1993; 21 : 1687-96.

15 Kusumoto FM, Mubiudeen IA, Kuecherer HF, Cabalan $M K$, Schiller NB. Response of the interatrial septum to transatrial pressure gradients and its potential for predicting pulmonary capillary wedge pressure: an intraoperative study using transesophageal echocardiography in patients during mechanical ventilation. JACC 1993; 21: 721-8.

16 Canadian consensus recommendations for the measurement and reporting of diastolic dysfunction by echocardiography. J Am Soc Echocardiogr 1996; 9: 736-60.

17 Gorcsan J III, Denault A, Gasior TA, et al. Rapid estimation of left ventricular contractility from end- systolic relations by echocardiographic automated border detection and femoral arterial pressure. Anesthesiology 1994; 81: 553-62.

18 Clements $F$. Monitoring ventricular function in the operating room. In: Otto $\mathrm{CM}$. The Practice of Clinical Echocardiography. Philadelphia: WB Saunders, 1997: 167-78.

19 Nomura T, Lebowitz L, Koide $\Upsilon$, Keehn L, Oka $\Upsilon$.

Evaluation of hepatic venous flow using transesophageal echocardiography in coronary artery bypass surgery: an index of right ventricular function. J Cardiothor Vasc Anesth 1995; 9: 9-17.

20 Van Daele ME, Sutherland GR, Mitchell MM, et al. Do changes in pulmonary capillary wedge pressure adequately reflect myocardial ischaemia during anaesthesia? A correlative preoperative hemodynamic, electrocardiographic, and transesophageal echocardiographic study. Circulation 1990; 81: 865-71.

21 Clements FM, de Bruijn NP. Perioperative evaluation of regional wall motion by transesophageal two-dimensional echocardiography. Anesth Analg 1987; 66: 249-61.

22 Shah PM, Kyo S, Matsumura $M$, Omoto $R$. Utility of biplane transesophageal echocardiography in left ventricular wall-motion analysis. J Cardiothor Vasc Anesth 1991; 5: 316-9.

23 Rouine-Rapp K, Ionescu P, Balea M, Foster E, Cabalan $M K$. Detection of intraoperative segmental wall- motion abnormalities by transesophageal echocardiography: the incremental value of additional cross sections in the transverse and longitudinal planes. Anesth Analg 1996; 83: 1141-8.

24 Leung JM, O'Kelly B, Browner WS, Tubau J, Hollenberg $M$, Mangano DT. Prognostic importance of postbypass regional wall-motion abnormalities in patients undergoing coronary artery bypass graft surgery. Anesthesiology 1989; 71: 16-25.
25 Comunale ME, Body SC, Ley C, et al. The concordance of intraoperative left ventricular wall-motion abnormalities and electrocardiographic S-T segment changes: association with outcome after coronary revascularization. Anesthesiology 1998; 88: 945-54.

26 London MJ, Tubau JF, Wong MG, et al. The "natural history" of segmental wall motion abnormalities in patients undergoing noncardiac surgery. Anesthesiology 1990; 73: 644-55.

27 Kato $M$, Nakashima $\Upsilon$, Levine J, Goldiner PL, Oka $\Upsilon$. Does transesophageal echocardiography improve postoperative outcome in patients undergoing coronary artery bypass surgery? J Cardiothor Vasc Anesth 1993; 7: 285-9.

28 Eisenberg $M J$, London MJ, Leung JM, et al. Monitoring for myocardial ischemia during noncardiac surgery. A technology assessment of transesophageal echocardiography and 12-lead electocardiography. JAMA 1992; 268: 210-6.

29 Pearlman AS, Gardin JM, Martin RP, et al. Guidelines for physician training in transesophageal echocardiography. Recommendations of the American Society of Echocardiography Committee for Physician Training in Echocardiography. J Am Soc Echocardiogr 1992; 5: 187-94. 